\title{
PSICOLOGÍA SOCIAL E IDENTIDAD: DIFICULTADES PARA UN ANÁLISIS PSICOSOCIOLÓGICO
}

\section{Social Psychology and identity: Difficulties for a psychosociological analysis}

\author{
Anastasio Ovejero* \\ * Universidad de Valladolid \\ tasio@psi.uva.es
}

\section{Palabras clave}

Psicología social Interdisciplinariedad Identidad Antipositivismo

\section{Resumen}

La época dorada de la Psicología Social tuvo mucho que ver con su interdisciplinariedad. Pero el positivismo fue haciendo que los psicólogos sociales colaboraran cada vez menos con otros científicos sociales, hasta el punto de que muchos de ellos hasta dejaron de sentirse científicos sociales. A esto se ha unido la progresiva sustitución de las explicaciones psicosociológicas por las meramente económicas a la hora de dar cuenta de la conducta humana e incluso de los hechos sociales. Y ambas cosas juntas han influido mucho en el actual declive de la psicología social como ciencia social. Ello se observa, por ejemplo, en la escasez de estudios realmente psicosociológicos sobre la identidad, un tema que debería ser central en esta disciplina. Por tanto, la psicología social debería hacer fundamentalmente dos cosas para recobrar la posición relevante que tenía entre las ciencias sociales: en primer lugar, hacerle frente a las propuestas economicistas y analizar cómo los procesos macrosociales conforman tanto la personalidad humana como la propia identidad, superando así el énfasis que actualmente se está poniendo en la identidad como un concepto meramente individual; y en segundo lugar, abandonar el positivismo que tanto daño está haciéndole a la psicología social y que constituye la verdadera traba para convertirse en una disciplina socialmente relevante. El concepto de identidad es uno de los más psicosociales de nuestra disciplina, es decir, es un concepto que une muy estrechamente lo psicológico y subjetivo, por una parte, y lo social y colectivo, por otra. Por eso la psicología social necesita imprescindiblemente un enfoque interdisciplinar que el positivismo hace muy difícil.

\section{Abstract}

The "golden age" of social psychology was related with its interdisciplinarity. But when social psychology adopted positivism, interdisciplinarity and "golden age" went away. Moreover, the rise of economics, not as a social science but a science of free market, has replaced social psychology for explain the human behavior and the social actions. If social psychology wants to recover its lost relevant position within the social sciences, it must make these two things: first, to face the economics explanations of human behavior, and analyze how macrosocial processes constitute the human personality and the human identity; and second, to leave behind its positivism to analyze the psychosociological processes and concepts, as is the case of human identity. The identity concept is necessary a psychosocial concept, a concept that narrowly connect the subjective and psychological, on the one hand, and the social and collective, on the other hand. Because of this, social psychology needs an interdisciplinary approach, approach that will possible only if the discipline abandon the positivism.

\section{Keywords}

Social Psychology Interdisciplinarity Identity Antipositivism
Ovejero, A. 2015, "Psicología Social e identidad: dificultades para un análisis psicosociológico", en Papeles del CEIC, vol. 2015/2, no 124, CEIC (Centro de Estudios sobre la Identidad Colectiva), Universidad del País Vasco, http://dx.doi.org/10.1387/pceic. 14314 


\section{INTRODUCCIÓN}

Existen básicamente tres niveles de aproximación a la conducta humana; individual, grupal y colectivo. Y el objetivo esencial de la psicología social consiste en interconectar esos tres niveles, que de hecho están siempre inextricablemente unidos. Individuo y sociedad son las dos caras de una misma moneda. Por eso resulta imposible aislar ninguna conducta humana, por individual que pueda parecer, del nivel grupal o del colectivo. Si queremos entender la conducta humana por fuerza deberemos acudir a esos tres niveles, pero considerándolos siempre como interdependientes e inseparables. La psicología social consiste esencialmente en conectar de diferentes maneras al individuo (o los procesos psicológicos individuales) con la sociedad (o los procesos sociales, tanto micro como macrosociales), de forma que los tres niveles de que hablaba puedan ser adecuadamente conectados. Y por eso la interdisciplinariedad debería caracterizar inevitablemente a esta disciplina, sobre todo en temas que, como es el caso de la identidad, exijan el análisis conjunto de esos tres niveles. De hecho, ya desde sus inicios la psicología social se nutrió tanto de la psicología como de la sociología, surgiendo tempranamente dentro de su seno dos psicologías sociales: una más psicológica y positivista y otra más sociológica, de las que la primera fue siempre la dominante. Ello dio lugar a una permanente tensión interna que se "resolvió" con una huida hacia adelante, olvidándose cada vez más de sus origenes y contenidos sociológicos, y afianzándose cada vez más su psicologismo y su positivismo, lo que está teniendo, a mi juicio, al menos estas cinco consecuencias: 1) un aislamiento cada vez mayor de las demás ciencias sociales; 2) una progresiva incapacidad para interconectar los tres niveles mencionados; 3) una gran dificultad para conseguir suficiente interdisciplinariedad; 4) una casi permanente ausencia de relevancia social; y 5) una pérdida de hegemonía dentro de las ciencias sociales. Con respecto a este último punto, recordemos, con House (2008), que si en 1970 los psicólogos sociales representaban el $18,50 \%$ de los socios de la ASA, en 2006 sólo representaban el 2,70\%; y si en 1970 el $68,80 \%$ de los 30 departamentos de sociología más importantes de EE. UU. tenían una especialidad en psicología social, en 2006 sólo la tenían el 34,20\%. De hecho, cuando más relevante fue nuestra disciplina y cuando su trabajo fue más fructífero fue precisamente cuando ambas ramas de la psicología social colaboraron y cuando, juntas, trabajaron también con 
otras disciplinas sociales (Sewell, 1989): su relevancia llegó incluso al punto de influir definitivamente en la decisión que en 1954 tomó la Corte Suprema estadounidense de suprimir la discriminación racial en las escuelas públicas.

\subsection{Declive de la psicología social}

Aunque la psicología social ha seguido la estela positivista desde $\mathrm{F}$. Allport (1924), y aunque no son nuevos los ataques internos a la disciplina por su falta de relevancia social (Ring, 1967), tal tendencia positivista se está afirmando cada vez más. El positivismo ha conseguido que sólo hablemos de cosas más o menos triviales, pero pocas veces relevantes. De ahí que la "época dorada" de la psicología social ha pasado ya, y no será fácil que vuelva si no cambiamos drásticamente la trayectoria de nuestra disciplina. Necesitamos volver a poner la interdisciplinariedad en el centro de nuestros objetivos y sobre todo de nuestra práctica como científicos sociales. Pero para ello es imprescindible abandonar definitivamente el positivismo. Algo parecido decía no hace mucho James S. House (2008): los años 1945-1970 vieron la "época dorada" de la ciencia social estadounidense en gran medida gracias al empuje precisamente de la psicología social. En cambio durante los últimos cuarenta años estamos asistiendo a un período de declive de la ciencia social empujado también por el propio declive de la psicología social, que ha sido sustituida por la economía a la hora de analizar los procesos sociales. No es por azar que los años 1945-1975 fueran los años de la socialdemocracia y a partir de 1975 haya sido un duro neoliberalismo conservador el que se ha implantado ya no sólo en las relaciones económicas y laborales, sino incluso en las relaciones interpersonales e intergrupales, justamente desde que se intentó, con tantos instrumentos a su servicio, que el famoso "Informe de la Trilateral" (Crozier, Huntington y Watanuki, 1975) se hiciera más y más real (véase Monedero, 2012). De ahí que si queremos que la psicología social vuelva a ocupar un lugar relevante dentro de las ciencias sociales deberíamos dedicar más tiempo y más esfuerzo a analizar los problemas reales del mundo actual, sobre todo aquellos que más afectan a la gente (véase Ovejero, 2014, 2015). Pero para ello es imprescindible abandonar el positivismo y adoptar nuevos enfoques psicosociológicos (Ovejero, 1999), y hacerlo desde una posición crítica (véase Ibáñez e İ̃̃iguez, 1997; Ovejero y Ramos, 2011).

Ese declive de la psicología social más sociológica a la vez que el progresivo auge de la más psicologista tiene que ver con una serie de factores que se están produciendo a lo largo de las últimas décadas, muy relacionados con el actual dominio del neoliberalismo tanto a nivel 
económico como a nivel político e ideológico (véase Ovejero, 2014, 2015): las explicaciones economicistas están sustituyendo a las explicaciones psicosociológicas a la hora de dar cuenta de la realidad social y sobre todo a la hora de construir la subjetividad. Probablemente sea la construcción del "sujeto neoliberal" el principal factor que actualmente está haciendo posible los cambios tan drásticos -y a mi juicio tan dañinos- que están teniendo lugar tanto en el campo económico como en el social (Laval y Dardot, 2013; Lazzarato, 2013; Ovejero, 2015). El neoliberalismo está consiguiendo que todos seamos, en mayor o menor medida, neoliberales, y con ello está cambiando incluso nuestra identidad. De ahí la importancia crucial que debería tener hoy día un análisis profundo e interdisciplinar de la identidad.

\subsection{Concepto de identidad}

De identidad dice Pérez Agote (1998: 366) en el Diccionario de Sociología de Giner, Lamo y Torres que aunque es un término antiguo que hace referencia a la mismeidad y a la continuidad de algo, en contraposición a la variedad y al cambio, no fue hasta el siglo $X X$ cuando se popularizó su uso y se introdujo en el ámbito de las ciencias sociales, habiendo sido en la psicología y en la sociología donde más protagonismo ha tenido, en la primera de la mano del psicoanálisis y con un sesgo individualista (Erikson, 1968), y en la segunda de la mano del interaccionismo simbólico, como algo esencialmente interactivo, es decir, psicosocial (Mead, 1934). Pero fue a partir de los años setenta, añade Pérez Agote, cuando se constituyó en Europa un nuevo campo de análisis social, el de la identidad colectiva (véase Ovejero, 1997), a partir de los análisis antropológicos de la etnicidad, de los análisis psicosociales de las relaciones intergrupales y de los análisis sociológicos del nacionalismo.

Pero si desde hace mucho se viene dando en Occidente una fuerte tensión entre la identidad personal y la colectiva, cada vez más se está resolviendo esa tensión a favor de la primera. En efecto, como escribe Elias:

"Es característico de la estructura de las sociedades más desarrolladas de nuestros días que el ser humano particular conceda más valor a aquello que le diferencia de otros, a su identidad como yo, que a aquello que tiene en común con otros, a su identidad como nosotros. Pero este tipo de equilibrio entre el yo y el nosotros, su decidida inclinación a favor del yo, es cualquier cosa menos evidente" (1990: 180). 
De hecho, aunque la identidad de las personas está más vinculada en las sociedades menos desarrolladas a la familia y al lugar del nacimiento y en las más desarrolladas a las decisiones y los éxitos personales, sin embargo en todas ellas existen los dos tipos de identidad, pues "Ia identidad como yo no puede existir sin la identidad como nosotros. Lo único que varía es el equilibrio entre el yo y el nosotros, la forma de la relación entre el yo y el nosotros" (Elias, 1990: 212), equilibrio que se está rompiendo en la actual sociedad neoliberal a favor del yo.

Por otra parte, ha sido el enfoque positivista dominante en la disciplina, unido al auge de las explicaciones economicistas y al retroceso de las psicosociológicas, sobre todo las interaccionistas (Howard, 2007; McCall, 2006), lo que ha llevado a la psicología social a estudiar la identidad mucho menos de lo que debiera, a pesar de tratarse de un concepto que por fuerza debería ser uno de los más centrales de la disciplina. Y es que la sociología y la psicología social están siendo sustituidas desde hace dos o tres décadas, a mi juicio muy peligrosamente, por la economía a la hora de explicar la conducta humana e incluso los procesos sociales. Por tanto, la psicología social debería hacerle frente a las propuestas economicistas y analizar cómo los procesos macrosociales conforman la personalidad humana (House, 1981) y la propia identidad (Wiegert, Teitge y Teitge, 1986), superando el énfasis que actualmente se está poniendo en la identidad como un concepto meramente individual. Como dice House: hoy día necesitamos "conceptos, teorías y métodos de psicología social sociológica e interdisciplinaria para complementar y equilibrar los conceptos, teorías y métodos actualmente más dominantes de la economía, la genética y la neurociencia cognitiva" (2008: 251). La simplicidad de los conceptos, las teorías y los métodos de la psicología social positivista no es capaz de captar la complejidad del concepto de identidad, un concepto tan relacional que por fuerza exige un esfuerzo interdisciplinar. La psicología social es en sí misma, como su propio nombre indica, una disciplina interdisciplinar, una disciplina psico-sociológica. También la identidad es un concepto a la vez individual y social. Por eso precisamente, y por la importancia que la situación tiene en la conducta humana (Ross y Nisbett, 1991), la identidad individual deriva de la identidad social, al igual que la propia personalidad deriva de -y refleja- la estructura social (House, 1981; Inkeles, 1959; Torregrosa y Fernández-Villanueva, 1984). 
El concepto de identidad es uno de los conceptos más psicosociales que existen, es decir, un concepto en el que se unen lo psicológico y subjetivo, por un lado, y lo social y colectivo, por otro, como se observa en análisis psicosociológicos de autores como Elias, Castells, Kelman o Torregrosa, análisis que tienen en cuenta a la vez su dimensión subjetivo-psicológica y su dimensión colectivo-social. La identidad y la pertenencia, con la que tan unida está, deberían constituir dos de los temas más centrales de la psicología social, dado que se trata de las dos necesidades más importantes del ser humano (véase Ovejero, 2010). Y es que la propia individualidad de cada persona es constitutivamente social. Como dice Elias:
"el ser humano individual vive, y ha vivido desde pequeño, dentro de una red de interdependencias que él no puede modificar ni romper a voluntad sino en tanto lo permite la propia estructura de esa red; vive dentro de un tejido de relaciones móviles que, al menos en parte, se han depositado sobre él dando forma a su carácter personal. Y en esto radica el verdadero problema: este contexto funcional posee una estructura muy específica en cada grupo humano" (1990: 29).

Y ello contribuye poderosamente a conformar nuestra memoria, y con ella nuestra identidad, que por tanto no serán meramente individuales, sino indiscutiblemente colectivas. Porque, añade Elias, "la individualidad que alcanzará finalmente una persona no depende simplemente de su constitución natural, sino de todo el proceso de individualización" (1990: 38), que es tanto como decir de socialización. El individuo humano no sería nada al margen de los procesos de socialización en que se formó. Sin esos procesos no seríamos sino el organismo meramente biológico que éramos al nacer. Nos fuimos haciendo personas precisamente a través de la interacción social dentro de los múltiples procesos en que nos fuimos socializando (socialización familiar, laboral, cultural, etc.). De ahí que "el carácter social del ser humano sólo podrá ser visto en su totalidad cuando se comprenda verdaderamente qué significan para el niño pequeño las relaciones con otras personas" (Elias, 1990: 41-42). Por tanto, concluye Elias: "las estructuras de la psique humana, las estructuras de la sociedad humana y las estructuras de la historia humana son fenómenos complementarios e inseparables, y sólo pueden ser estudiados dentro de un contexto que abarque sus relaciones mutuas" (1990: 54). Por eso cada individuo es único y distinto de todos los demás, porque ningún otro ha estado en los mismos contextos 
sociales ni ha sido socializado dentro de las mismas relaciones sociales, porque, en definitiva, no ha vivido las mismas interacciones sociales.

Ahora bien, dado que los tipos de socialización han ido cambiando a lo largo de la historia, también han ido cambiando los tipos de identidad. Así, Hall (1992) habla de tres grandes clases de identidad. La primera, la de la llustración, era muy genérica y veía al ser humano como un ser universal, autocentrado y provisto de la capacidad de la razón. La segunda, la de la Sociología, refleja ya la creciente complejidad del mundo moderno y toma conciencia de que su unidad interna no resulta suficiente, lo que lleva, necesariamente, a una concepción interactiva de la identidad, que es la que desarrolló G.H. Mead y el interaccionismo simbólico. Finalmente está la identidad del sujeto postmoderno, que es una identidad esencialmente fragmentada y caracterizada por la falta de estabilidad. Hoy día todo es líquido, también la identidad. La incertidumbre está en el centro de nuestra época y ello influye por fuerza y de una forma crucial en nuestra identidad. De ahí las enormes dificultades que la gente tiene hoy día para construirse una identidad que, por definición, debería tener al menos un cierto grado de estabilidad (Bauman, 2005; Beck, 1992; Giddens, 1993, 1995).

\section{FRAMENTACIÓN DE LA IDENTIDAD EN LA ÉPOCA ACTUAL}

Para Habermas, y por decirlo con palabras de Honnetth, "Ios seres humanos son capaces de desarrollar identidades personales sólo en la medida en que pueden crecer dentro de un mundo intersubjetivo compartido de un grupo social y moverse dentro de él" (2009: 329). Y eso es justamente lo que está desapareciendo en la actual Postmodernidad (véase Ovejero, 1999). De hecho, en la sociedad occidental fue la Modernidad la que definió con más claridad la identidad, tanto la individual como la colectiva, en especial la derivada de la nación, pero últimamente se han fragmentado y hecho añicos ambas identidades. Ello se debe a varios factores entre los que está el hecho de que, como escribe Castells (2006), en la actual globalización neoliberal los valores económicos se imponen a las personas sin que estas tengan mucha capacidad de controlarlos, ni siquiera con su voto colectivo, lo que lleva a mucha gente a buscar su identidad nuevamente en su dios, su religión, su patria o su equipo de fútbol. Además, a buena parte de la población le están impidiendo hacer proyectos de vida, con lo que millones personas, 
especialmente jóvenes, desempleadas o con contratos muy precarios y sin futuro ninguno, están teniendo serios problemas de identidad, no pudiendo ni siquiera forjarse la identidad de ciudadanía que hasta hace muy poco definía a la clase trabajadora europea (Crespo, Prieto y Serrano, 2009). Por tanto, son dos los factores más responsables de la actual fragmentación de las identidades:

A) La Postmodernidad: con el final de las certidumbres termina también una época segura para las identidades. Ya Fromm (1967) abordaba el tema de la identidad desde su concepto del carácter social, argumentando que cada época construye un hombre portador de los valores centrales del sistema y que lo hace a través de los procesos de socialización. Así, el feudalismo hizo hombres sumisos ante la autoridad y muy respetuosos con ella y con las jerarquias sociales, que es lo que necesitaban entonces los poderosos; la revolución industrial produjo un sujeto motivado hacia el trabajo y hacia el beneficio económico, disciplinado, puntual y ahorrador; el capitalismo postbélico construyó un hombre motivado esencialmente hacia el consumo; y el actual capitalismo neoliberal está produciendo un ser humano con una identidad fragmentada y líquida, de forma que esta depende en gran medida de su éxito personal, un éxito a menudo ajeno a él mismo. Y es que en esta Postmodernidad nada es ya para siempre, sino que todo es líquido, incluso la identidad, lo que lleva a un evidente nomadismo identitario.

Y por si todo lo anterior fuera poco, está también la influencia de los medios de comunicación que en gran medida controlan la identidad de las personas y de los grupos humanos. Como afirma Sampedro Blanco:

\footnotetext{
"Los medios de comunicación nos transmiten representaciones y mensajes de quiénes somos y a qué grupo pertenecemos. A partir de su recepción se asientan la identidad individual (nuestras características y potencialidades, enmarcadas en una trayectoria vital) y la identidad colectiva (sentido de pertenencia a un grupo con un pasado y un futuro comunes). Ambas identidades se consolidan y transforman en nuestras interacciones diarias, con otros individuos y colectivos que reconocemos como diferentes" (2003: 9).
}

De ahí que también Internet esté fragmentando nuestra identidad, pues, como subraya Rendueles, "la participación en el entorno tecnológico es 
el vector que unifica la plasticidad extrema de nuestra propia identidad" (2013: 91).

B) Nuevo capitalismo neoliberal: dado que tanto la estructura social como el lugar que cada grupo y cada individuo ocupan en esa estructura determinan la identidad de los grupos y de los individuos humanos, no es de extrañar que las políticas neoliberales estén influyendo mucho en la formación de la identidad, en el sentido de una cada vez mayor dificultad para que la ciudadanía, y sobre todo los trabajadores, puedan construirse una identidad estable. De hecho, "el mercado de las identidades tiende a reproducir las desigualdades estructurales de los individuos y los grupos sociales, que pugnan (con desiguales recursos materiales y simbólicos) para hacerse con unas identidades públicas que hagan avanzar sus intereses" (Sampedro Blanco, 2003: 10). Y entre las muchas características del nuevo capitalismo neoliberal quisiera destacar tres que producen muy serios problemas de identidad en muchos millones de personas:

a) Incremento de las desigualdades: entre sus numerosos efectos para los ciudadanos está el daño producido a su identidad, tanto personal como colectiva (Bauman, 2014; Wilkinson, 2001; Wilkinson y Pickett, 2009).

b) Consumismo: el énfasis que el actual capitalismo pone en la capacidad de consumo como principal pilar de la identidad lleva por fuerza a serios problemas de identidad a todas aquellas personas que no pueden consumir (desempleados...).

c) La devastación del mundo del trabajo: el nuevo capitalismo, a través sobre todo de una auténtica devastación del trabajo (Ovejero, 2014), está generando "la experiencia de un tiempo desarticulado que amenaza la capacidad de la gente de consolidar su carácter en narraciones duraderas" (Sennett, 2000: 30), lo que conlleva muy serias dificultades para la construcción de una identidad tanto personal como colectiva. No otra cosa es, a mi modo de ver, la "corrosión del carácter" de que habla Sennett.

La consecuencia de todo ello es un profundo nomadismo identitario. En efecto:

"la perspectiva de la migración profesional, de la migración territorial, de la migración competencial y de la inseguridad de las retribuciones, alimentan un imaginario donde las identidades estables, duraderas, y, más aún, las identidades 
permanentes configuradas en base al trabajo, dejan de tener sentido. Fin, por lo tanto, no del trabajo, pero sí de esa peculiar ideología del trabajo que fue tan importante en la última fase de la modernidad; y fin, también, de lo que podríamos llamar el sedentarismo identitario, sustituido por la perspectiva de un nomadismo identitario" (Ibáñez, 2014: 106).

Por tanto, "la necesidad de adaptación continua al cambio, la exposición constante a nuestros paradigmas y contingencias globales y locales hará más intensas las fuerzas que fragmentan la identidad provocando una mayor incidencia de problemas psicológicos en el trabajo" (De Venanzi, 2002: 310). En consecuencia, añade este autor:

“la organización y funcionamiento de un sistema así sustentado conlleva la aparición de un ciudadano que poco a poco se convierte en pasivo y apático. Es un individuo a quien le resulta difícil establecer vínculos sociales significativos más allá de la familia y desarrollar una identidad que trascienda la abrumadora fuerza estética del consumo" (2002: 316-317).

Es el sujeto neoliberal, principal producto del nuevo capitalismo y, a la vez, su principal sostén (Laval y Dardot, 2013; Lazzarato, 2013; Ovejero, 2015). La explicación podría ser la siguiente: como es sabido, para Foucault el sometimiento material constituye un aspecto esencial del proceso de constitución del sujeto. Más en concreto, para él el poder es algo que no sólo reprime sino que también crea, de manera que contribuye poderosamente a formar incluso al sujeto que se le opone:

"El modelo habitual para entender este proceso es el
siguiente: el poder nos es impuesto y, debilitados por su
fuerza, acabamos internalizando o aceptando sus
condiciones. Lo que esta descripción omite, sin embargo, es
que el 'nosotros' que acepta esas condiciones depende de
manera esencial de ellas para 'nuestra existencia'" (Butler,
$2011: 12$ ).

Sin embargo, Foucault no desarrolló ni explicitó los mecanismos específicos que subyacen a este proceso, es decir, los mecanismos por los que el sujeto se forma en la sumisión. Estos mecanismos pueden atisbarse en la teoría del amo y el esclavo de Hegel, según la cual el amo, que al principio es externo al esclavo, reemerge más tarde como la propia conciencia de este. "En todos los casos, el poder que en un principio aparece como externo, presionando sobre el sujeto, 
presionando al sujeto a la subordinación, asume una forma psíquica que constituye la identidad del sujeto" (Butler, 2011: 13).

Pero la identidad que las personas tenían durante la modernidad ha sido hecha añicos tanto por la postmodernidad como por la globalización neoliberal y sus prácticas laborales (véase Ovejero, 2014). Más aún, como escribe De Venanci (2002: 98), las acciones y decisiones implican ahora enfrentar dilemas y riesgos, en tanto los procesos de individualización y de construcción de identidades se vuelve altamente compleja en virtud de la variabilidad de modelos socioculturales novedosos e inesperados a disposición de los hombres (Beck, 1992). Pero no es sólo la "flexibilidad" (precarización e inseguridad laboral extrema) producida por la hegemonía neoliberal, sino también la propia individualización producida por la postmodernidad, lo que está haciendo cada vez más difícil la construcción de una identidad personal y colectiva satisfactorias. Más en concreto, añade De Venanzi:

"el individuo se ve obligado a revisar su identidad continuamente mediante un proceso reflexivo (consistente de una revisión crónica de todos los aspectos de la vida social a la luz de nueva información y conocimiento) de actualización; es decir, frente a muchas contingencias, crisis y dialécticas entre lo local/global, el hombre debe lograr una narrativa biográfica coherente" (2002: 131).

Cosa harto difícil en estas circunstancias. Claramente lo dice Giddens (1993: 147): "Vivimos en un tiempo en que la misma experiencia privada de tener que descubrir la identidad personal y de lograr un destino personal se ha convertido en una fuerza política subversiva de enormes proporciones".

En definitiva:

"la modernidad líquida es un entorno extremadamente hostil para quienes aspiran a desarrollar una identidad sólida, una subjetividad continua basada en una narrativa teleológica. El triunfador del turbocapitalismo es profundamente adaptativo: tiene distintos yoes, diversas personalidades familiares, ideológicas o laborales. Los perdedores también. Los trabajadores migrantes ya no se van a otro país con la idea de iniciar una nueva vida más próspera, sino que diseminan su fuerza de trabajo saltando de país en país siguiendo los caprichosos flujos financieros. Los terapeutas también nos exhortan a aceptar esta fluidez extrema. Quien 
se aferra a una identidad política, sentimental o moral ya no es ni siquiera un perdedor o un resentido, sino directamente un sujeto patológicamente poco adaptativo" (Rendueles, 2013: 181).

\section{CONCLUSIÓN}

La identidad es un concepto esencialmente psicosocial que, por tanto, debería constituir uno de los temas de estudio más centrales de la psicología social. Sin embargo, ello no ha sido así antes y lo es menos aún ahora. El positivismo de la psicología social dificultó siempre un abordaje adecuado al estudio de la identidad. Pero a esa dificultad se ha añadido durante las últimas décadas una más: la fragmentación de la propia identidad humana, lo que hace ya definitivamente inútil cualquier enfoque positivista en este campo: la única forma de que la psicología social pueda analizar con eficacia la identidad humana pasa inevitablemente por el abandono del positivismo, como desde hace años está proponiendo la nueva psicología social (Ovejero, 1999).

Más en concreto, la pérdida de relevancia social de la disciplina tiene mucho que ver con los cambios sociales que están teniendo lugar y que están llevando a que las explicaciones psicosociales sean sustituidas por las meramente economicistas a la hora de analizar conducta humana y los propios procesos sociales, pero tiene que ver también con su enfoque positivista. De ahí que la mayoría de los estudios existentes en nuestra disciplina sobre identidad (Erikson, Freud, Tajfel, Turner...) hablen de la identidad personal e incluso a veces de la grupal, pero no de la identidad colectiva, a pesar de que es de ella de la que extraemos nuestra identidad personal: la identidad colectiva es sin ninguna duda un asunto esencialmente psicosocial.

Estamos en el último peldaño, hasta ahora, del proceso de individualización que empezó hace unos ochocientos años: lo que ganamos en libertad lo perdemos en seguridad, como ya advirtiera Fromm (1941). Pero incluso la libertad nos resulta difícil de soportar, pues ahora ser libres se ha convertido en un deber más que en un derecho. Si en la Modernidad nuestra identidad en gran medida nos la hacían, ahora somos nosotros quienes tenemos que construirla, pero siempre y cuando las circunstancias (por ejemplo las laborales) nos lo permitan. Por eso habla Bauman (2005) de una "identidad flotante ". La identidad que nos exige la modernidad líquida es, por una parte, plural y abierta, mientras que, por otra parte, es flexible y, por consiguiente, muy frágil.

Según Zymunt Bauman, y por decirlo con palabras de Helena Béjar: 
"en la modernidad sólida, el problema era cómo conseguir una identidad adquirida a través de la determinación social (por la clase, el estatus o el rol) y 'cómo conservarlo y evitar el desalojo'. En la modernidad líquida, es qué identidad elegir y cómo adecuar los posibles cambios a la mudanza continua de las metas" (2007: 121).

Pero cuando no se tiene clara la propia identidad puede surgir lo que Maalouf (2005) Ilama identidades asesinas, que, siguiendo la estela de Muzafer Sherif, podemos evitar construyendo una identidad grupal común (Nier, Gaertner, Dovidio, Banker, Ward y Rust, 2001). De hecho, existe evidencia empírica que sugiere que:

\begin{abstract}
"el tener una identidad intragrupal común mediatiza los efectos de las variables de la hipótesis del contacto, incrementando los sentimientos y las conductas positivas hacia miembros concretos del exogrupo. Más aún, somos optimistas porque una identidad supraordinal puede iniciar unas interacciones más abiertas y personalizadas, así como unas orientaciones más cooperativas y altruistas hacia los miembros del exogrupo. Aunque la representación cognitiva de una identidad supraordinal pueda ser a menudo breve o inestable, parece que es capaz de iniciar conductas que piden reciprocidad $y$, de esta manera, puede tener consecuencias grupales más permanentes. También pueden algunas de estas conductas iniciar procesos que pueden reducir los sesgos a través de otras vías adicionales. Por ejemplo, consideramos que la principal ventaja de inducir una identidad común es su capacidad para cambiar temporalmente el curso de las interacciones intergrupales y de iniciar procesos e intercambios constructivos tanto intergrupales como interpersonales de una manera que tenga efectos más duraderos para producir unas relaciones más positivas entre los grupos" (Gaertner y Dovidio, 2009: 502).
\end{abstract}

Pero no resulta fácil estudiar adecuadamente estos temas sin abandonar el posititivismo y resulta difícil encontrar una identidad supraordinal en este capitalismo neoliberal.

Por último, quisiera añadir, para terminar, que toda reconstrucción de una psicología social que sea relevante socialmente y que sea capaz de abordar los temas psicosociales, como es el caso de la identidad, necesita algunos cambios esenciales, entre los que yo destacaría estos dos:

- Modificar radicalmente la concepción mecanicista del ser humano y, en consecuencia, abandonar los métodos tradicionales (sobre todo los experimentales). 
- Adoptar una mayor interdisciplinariedad, dada la artificialidad de las fronteras entre las ciencias sociales y dada la necesidad de que los temas psicosociales, como es el caso de la identidad, sean abordados conjuntamente por psicólogos sociales, sociólogos, antropólogos y otros científicos sociales.

\section{BIBLIOGRAFÍA}

Allport, F.H., 1924, Social psychology, Houghton and Mifflen, Boston.

Bauman, Z., 2005, Identidad, Losada, Buenos Aires.

Bauman, Z., 2014, ¿La riqueza de unos pocos nos beneficia a todos? Paidós, Barcelona.

Beck, U., 1992, La sociedad del riesgo, Paidós, Barcelona.

Béjar, H., 2007, Identidades inciertas: Zygmunt Bauman, Herder, Barcelona.

Butler, J., 2011, Mecanismos psíquicos del poder, Cátedra/Universidad de Valencia, Madrid.

Castells, M., 2006, En qué mundo vivimos. Conversaciones con Manuel Castells, Alianza, Madrid.

Crespo, E., Prieto, C., Serrano, A. (Eds.), 2009, Trabajo, subjetividad y ciudadania: Paradojas del empleo en una sociedad en transformación, Editorial Complutense y Centro de Investigaciones Sociológicas, Madrid.

Crozier, M.J., Huntington. S.P., Watanuki, J., 1975, The Crisis of Democracy. Report on the Governability of Democracies to The Trilateral Commission, New York University Press.

De Venanzi, A., 2002, Globalización y corporación. El orden social en el siglo XXI, Anthropos, Barcelona.

Elias, N., 1990, La sociedad de los individuos, Península, Barcelona.

Erikson, E.H., 1968, Identity, youth and crisis, W. W. Norton Company, New York.

Fromm, E., 1967, Psicoanálisis de la sociedad contemporánea, F.C.E., México.

Fromm, E., 1941, Escape from freedom, Henry Holt and Company, Nueva York.

Gaertner, S., Dovidio, J., 2009, "A common ingroup identity: A categorization-based approach for reducing intergrupo bias", en 
T.D. Nelson (Ed.), Handbook of prejudice, stereotyping, and discrimination, Psychology Press/Taylor and Grancis Group, Nueva York, pp. 489-505.

Giddens, A., 1993, Consecuencias de la modernidad, Alianza, Madrid.

Giddens, A., 1995, Modernidad e identidad del yo, Editorial 62, Barcelona.

Hall, S., 1992, "The question of cultural identity", en S. Hall et al. (Eds.), Modernity and its futures, Polity Press, Cambridge, pp. 274-325.

Honneth, A., 1997, La lucha por el reconocimiento: por una gramática moral de los conflictos sociales, Crítica, Barcelona.

House, J.S., 1981, "Social estructure and personality", en M. Rosenberg y R. Turner (Eds.), Sociological perspectives in social psychology, Basic Books, Nueva York, pp. 525-561.

House, J.S., 2008, "Social psychology, social science, and ecomimics: Twentieth century progress and problems, twenty-first century prospects", en Social Psychology Quarterly, 71(3), pp. 332-356.

Howard, J.A., 2007, "The state of (cumulative theoretical) social psychology", en Contemporary Sociology, 36(5), pp. 418-420.

Ibáñez, T., 2014, Anarquismo es movimiento, Virus, Barcelona.

Ibáñez, T., İñiguez, L. (Eds.), 1997, Critical Social Psychology, SAGE, London.

Inkeles, A., 1959, "Personality and social structure", en R.K. Merton, L. Broom y L.S. Cottrel. Jr., (Eds.), Sociology today, Nueva York, Basic Books, pp. 249-276.

Laval, C., Dardot, P., 2013, La nueva razón del mundo. Ensayo sobre la sociedad neoliberal, Gedisa, Barcelona.

Lazzarato, M., 2013, La fábrica del hombre endeudado, Amorrortu, Buenos Aires.

Maalouf, A., 2005, Identidades asesinas, Alianza, Madrid.

McCall, G.J., 2006, "Simbolic Interaction", en P.J. Burke (Ed.), Contemporary social psychological theories, Stanford University Press, Palo Alto, CA, pp. 1-23.

Monedero, J.C., 2012, "El programa de máximos del neoliberalismo: el Informe a la Trilateral de 19751", en Sociología Histórica, 1, pp. 298310.

Mead, G.M., 1972, Espiritu, persona y sociedad, Paidós, Buenos Aires. 
Nier, J.A., Gaertner, S.L., Dovidio, J.F., Banker, B.S., Ward, C.M., Rust, M.C., 2011, "Changing interratial evaluations and behavior: The effects of a common group identity", en Group Processes and Intergroup Relations, 4, pp. 299-316.

Ovejero, A., 1997, El individuo en la masa: Psicología del comportamiento colectivo, Ediciones Nobel, Oviedo.

Ovejero, A., 1999, La nueva psicología social y la actual postmodernidad, Servicio de Publicaciones de la Universidad de Oviedo, Oviedo.

Ovejero, A., 2010, Psicología social: Algunas claves para entender la conducta humana, Biblioteca Nueva, Madrid.

Ovejero, A., 2014, Los perdedores del nuevo capitalismo: Devastación del mundo del trabajo, Biblioteca Nueva, Madrid.

Ovejero, A., (en prensa), “Psicología Social y globalización neoliberal”, en M. Morales et al. (Eds.), s/t, Universidad Pontificia Bolivariana. Medellín.

Ovejero, A., Ramos, J., 2011, Psicología Social Crítica, Biblioteca Nueva, Madrid.

Pérez Agote, A., 1998, "Identidad”, en S. Giner, E. Lamo y C. Torres (Eds.), Diccionario de Sociología, Alianza, Madrid, p. 366.

Rendueles, C., 2013, Sociofobia: El cambio político en la era de la utopía digital, Editorial Capitán Swing, Madrid.

Ring, K.R., 1967, “Experimental social psychology: some sober questions about some frivolous values", en Journal of Experimental Social Psychology, 3, pp. 113-123.

Ross, L., Nisbett, R.E, 1991, The person and the situation, Temple University Press, Philadelphia.

Sampedro Blanco, V.F., 2003, "Medios de comunicación, políticas y mercados de identidad", en V.F. Sampedro Blanco (Ed.), La pantalla de las identidades: Medios de comunicación, políticas y mercados de identidad, Icaria, Barcelona, pp. 9-26.

Sennett, R., 2000, La corrosión del carácter. Las consecuencias personales del trabajo en el nuevo capitalismo, Anagrama, Barcelona.

Sewell, W.H., 1989, "Some reflections on the golden age of interdisciplinary social psychology", en Social Psychology Quarterly, 52(2), pp. 88-97.

Torregrosa, J.R., Fernández Villanueva, C., 1984, "La interiorización de la estructura social", en J.R. Torregrosa y E. Crespo (Eds.), Estudios 
básicos de psicología social, Editorial Hispano-Europea, Barcelona, pp. 421-426.

Wiegert, A.J., Teitge, J.S., Teitge, D.W., 1986, Identity and society: Toward a Sociological Psychology, Cambridge University Press, Cambridge.

Wilkinson, R., 2001, Las desigualdades perjudican: Jerarquías, salud y evolución humana, Crítica, Barcelona.

Wilkinson, R., Pickett, K., 2009, Desigualdad: Un análisis de la (in)felicidad colectiva, Turner, Madrid. 